\title{
Risk Mitigation for ITER by a Prolonged and Joint International Operation of JET
}

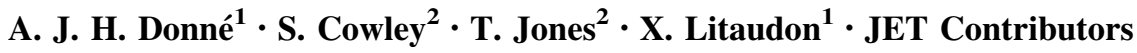

Published online: 9 September 2015

(c) The Author(s) 2015. This article is published with open access at Springerlink.com

\begin{abstract}
Prolonged operation of the Joint European Torus (JET) in a set-up involving all ITER partners will be beneficial for ITER. Experiments at JET with its ITER-like wall and using a D-T plasma mixture will help to mitigate risks in the ITER research plan. Training of the ITER operators, technicians and engineers at JET will safe valuable time when ITER comes into operation. Moreover, the way in which the future ITER experiments will be organized can already be experienced at JET, by imposing a similar organisational structure. This paper will present arguments in favour of an extension of JET and additionally briefly discuss a number of enhancements that will make experiments on JET even more relevant for ITER.
\end{abstract}

Keywords Nuclear fusion - Deuterium-tritium - JET . ITER - Electron cyclotron resonance heating - Resonant magnetic perturbations

\section{Introduction}

The present ITER time schedule [1] still has 2021 as the year of first plasma. However, it is general knowledge that the ITER construction is strongly delayed and therefore, the ITER schedule is being reviewed in order to minimize

For JET Contributors, see the Appendix of Romanelli et al. [6].

A. J. H. Donné

tony.donne@euro-fusion.org

1 Programme Management Unit, EUROfusion Consortium, JET, Culham Science Centre, Abingdon OX14 3DB, UK

2 Culham Centre for Fusion Energy, Culham Science Centre, Abingdon OX14 3DB, UK these delays. By the end of 2015 the ITER team will announce a new date for first plasma and also for D-T operation. According to the current schedule, ITER will start operation in hydrogen $(\mathrm{H})$ and helium $(\mathrm{He})$ in order to avoid the machine to become radioactive. After approximately 3-4 years, ITER will switch to operation with deuterium and finally, 7-8 years after first plasma, a mixture of deuterium (D) and tritium (T) will be used. During the hydrogen and helium phase, most ITER systems will be tested as far as possible. This should give an indication how these systems will function in plasmas with a D-T mixture.

Even though the focus of the international community is very much (as it should be) on the ITER construction, there is an additional need and benefit to exploit existing fusion facilities as effectively as possible so as to optimize the exploitation of ITER, to minimize risks in ITER operation and thereby to ensure a rapid transition to D-T operation of the ITER device. Furthermore, an understanding is developing that achieving efficient and productive operation of ITER implies not simply the implementation of relevant experiments in present fusion facilities, but also training of the future generation of scientists, technicians and engineers in the effective exploitation of a large nuclear fusion facility. This latter goal is likely to require the establishment of international task forces which would implement (ITER-related) experimental research programmes on existing fusion devices using organizational procedures appropriate to the implementation of research programmes on ITER and making use of operational tools (e.g. plasma control system), but also of the data analysis and modelling tools foreseen for ITER.

Since ITER will be a nuclear device, the way to prepare the experiments will be very different from how it is done in contemporary fusion devices, with much more emphasis on pulse preparation and modelling prior to the experiment. 
Although risk mitigation and training can be done with several of the main fusion devices in the world, we will address in this paper on how specifically the Joint European Torus, JET, can be utilized in the coming decade to mitigate risks in the ITER research plan by doing specific research and, to train the ITER control room team (operators, session leaders, technicians, etc.). Until ITER comes into operation, JET is the only device that can test with all three hydrogen isotopes and it can therefore help to understand how the plasma behaviour in hydrogen extrapolates to deuterium and tritium. Moreover, JET is the device closest to ITER as it has a number of unique features: it has an ITER-like wall and related beryllium-handling facilities; it can operate with deuterium-tritium plasmas; and it has tritium gas handling systems. Additionally, it is the only device that is almost fully remotely maintainable. Some of the JET subsystems could even be equipped with the same operating system as on ITER. JET is therefore an ideal device to train the ITER generation of scientists and engineers on how to operate a nuclear fusion device. JET can be used to perform ample experiments that will help to mitigate the risks in the ITER research plan.

Although this paper focuses on the impact and advantages that a prolonged operation of JET would bring as risk mitigation for ITER, it is important to mention that the presently approved reference schedule for JET runs until the end of 2018. Any extension of JET beyond that time frame will need approval of the European Commission, the government of the United Kingdom as well as the General Assembly of EUROfusion.

\section{JET_Past and Expected Performance}

In 1997 JET has used tritium to produce up to $16 \mathrm{MW}$ of fusion power during a series of dedicated experiments. This was an important result since for the first time conditions close to breakeven were obtained [2]: $16 \mathrm{MW}$ was produced with $25 \mathrm{MW}$ injected into the reaction chamber $\left(\mathrm{Q}=\mathrm{P}_{\text {fusion }} / \mathrm{P}_{\text {input }} \cong 0.6\right)$. However, these conditions were maintained only for $<1 \mathrm{~s}$. In stationary conditions a total of $4 \mathrm{MW}$ were produced [3], at an input power of $22 \mathrm{MW}$ (hence $\mathrm{Q} \cong 0.18$ ). This was done during $5 \mathrm{~s}$, which is longer than the characteristic thermal confinement time scales of the plasma processes. During these JET D-T experiments (referred to as DTE1) a few unexpected (and still unexplained) phenomena were discovered. For example, the transition to plasma regimes with reduced turbulence level was achieved more easily; very good news for ITER. However, due to the limit on the total number of D$\mathrm{T}$ fusion reactions that were allowed at that time, the investigation was restricted to a standard regime of operation developed on JET. The advanced regime with an
Internal Transport Barrier, the so called optimised shear regime, was also attempted [4] but it was not fully developed in D-T. It turned out that the transferability of the scenario from $\mathrm{D}$ to $\mathrm{D}-\mathrm{T}$ is not straightforward since the $\mathrm{L}$ to $\mathrm{H}$ threshold is different, and hence, the q-profile evolution and the scenario need to be re-tuned. In this respect it is important to note that the ITER hybrid scenario for long pulse operation was not at all investigated; so there could be similar unexpected hurdles in developing this scenario for D-T.

Since 2011, JET operates with a full metal wall made out of beryllium and tungsten $[5,6]$. Equipped with the same wall materials as foreseen for ITER ('ITER-like wall'), JET has basically become a small version of ITER, which can gain experience with $\mathrm{D}-\mathrm{T}$ experiments in an ITER-like environment and with ITER-relevant plasma scenarios. Doing D-T experiments in JET to prepare ITER's operation will lower the risk for further ITER delays and related cost increases. On the long run it will reduce the time needed for ITER to achieve its main goal, which is to demonstrate the technological and scientific feasibility of fusion energy at $Q=10$. If an obstacle is discovered today on JET there is enough time to develop alternative strategies. An obstacle discovered on ITER would immediately delay its exploitation and increase its costs.

Already it was recently found on JET that the operating conditions imposed by the metallic wall materials lead to a significant re-optimisation of the plasma scenarios to reach a similar level of fusion performance and thermal energy content as previously observed for similar operational parameters (toroidal field, plasma current, applied power) but with the C-wall. With the available applied power in the range of 26-30 MW (consisting typically of 22-27 MW of neutral beam power and $4 \mathrm{MW}$ of ICRH power), the JET performance has been recovered in 2014 up to a plasma current of 2.5MA for both the ITER baseline $\left(\beta_{\mathrm{N}} \sim 2\right)$ and hybrid scenarios $\left(\beta_{\mathrm{N}} \sim 2.3-3.0\right)$, with on-axis safety factor above unity. As part of the scenario development to increase the fusion performance it is essential that attention is devoted to minimize the occurrence of disruptions. That is applying disruption avoidance techniques, reducing the plasma disruptivity, and thus minimizing the consequences to the device. It is recalled that inadequate disruption mitigation is the highest programmatic risk in the ITER research plan. The dynamics of the unmitigated disruptions with the ILW is found to be different with respect to the C-wall [7]. In this context, it was found that mitigation by Massive Gas Injection became a necessity for ILW operations at plasma currents higher than 2.0 MA [8].

Twenty years of experiments world-wide have led to improved regimes of operation for ITER that need to be 
tested in D-T on JET with plasma parameters as close as possible to those in ITER. Many of these regimes have been tested in $\mathrm{H}$ and $\mathrm{D}$. As mentioned above it is not straightforward to transfer plasma scenarios from $\mathrm{H}$ or $\mathrm{D}$ to other isotope mixtures (as full tritium: T-T, or deuteriumtritium: D-T). Therefore, the high level objectives of a future JET D-T campaign should include the repeat of scenarios developed with other gas mixtures or in other machines to ensure that these also work with a combination of an ITER-like wall and a D-T mixture. It is unlikely that scenarios developed in a carbon wall machine can be straightforwardly transferred to ITER, as a metal wall has a large impact on the detailed plasma processes.

A D-T campaign should therefore include the following high level objectives:

- Explore the scenarios foreseen in ITER with emphasis on core and edge integration, and transferability from $\mathrm{H}$ and $\mathrm{D}$ to $\mathrm{T}-\mathrm{T}$ and $\mathrm{D}-\mathrm{T}$ plasmas;

- Study the isotope effect and complete research done in $\mathrm{H}$ and $\mathrm{D}$ to $\mathrm{T}-\mathrm{T}$ and $\mathrm{D}-\mathrm{T}$;

- Get experience with the tritium cycle and handling and in particular with fuelling, retention, migration, recovery, and dust. This can be done in $\mathrm{T}-\mathrm{T}$ and $\mathrm{D}-\mathrm{T}$ plasmas;

- Study $\alpha$-particle physics in conditions close to the burning plasma regime in D-T: study the impact of the $\alpha$-particles on confinement and stability;

- Get experience with neutronics in D-T: diagnostics calibration, behaviour of materials under high neutron flux and fluence, code validation.

The performance target in order to study these high level objectives is to achieve a stationary fusion plasma with ITER-like wall (ILW) and with $\mathrm{W}_{\text {fusion }} \sim 50-75 \mathrm{MJ}, \mathrm{P}_{\mathrm{fu}}$ sion $\sim 10-15 \mathrm{MW}$ for $5 \mathrm{~s}$. This would be roughly consistent with the physics assumptions used for ITER in estimating its baseline performance (see Table 1). The combined achievement of such a high fusion performance in a device with an ITER-like wall in JET would increase confidence in successful ITER operation with the same combination of plasma facing components: tungsten in the divertor and beryllium in the plasma facing components, despite the fact that the implementation of the plasma facing components is different on JET, which is a shortpulse device without active cooling.

An advanced regime of operation (the so-called hybrid regime) has been developed on JET in recent years [6]. In this regime the confinement of energy inside JET is increased up to $\mathrm{H} \sim 1.2-1.3$. This JET regime has thus far been achieved only up to a plasma current of 2.5 MA with $26 \mathrm{MW}$ of input power at $\mathrm{H} \sim 1.1$ for a duration of $0.5 \mathrm{~s}$. If it can be extended at higher power (40 MW), higher plasma current (in the range of 2.5-3 MA) and longer
Table 1 Comparison of JET's "ITER baseline" scenario with planned ITER 15MA

\begin{tabular}{lll}
\hline Parameter & $\begin{array}{l}\text { JET (ITER } \\
\text { baseline) } \\
\text { extrapolated } \\
\mathrm{H}_{98} \sim 1.0\end{array}$ & $\begin{array}{l}\text { ITER (1 5 MA) } \\
\text { baseline }\end{array}$ \\
& $\begin{array}{l}\mathrm{H}_{98} \sim 1.0 \\
2.89 / 0.94\end{array}$ & $6.2 / 2.0$ \\
\hline $\mathrm{R} / \mathrm{a}[\mathrm{m} / \mathrm{m}]$ & $3.65(3.85)$ & 5.3 \\
Toroidal field $\mathrm{B}_{\mathrm{T}}[\mathrm{T}]$ & $4(4.5)$ & 15.0 \\
Plasma current $[\mathrm{MA}]$ & 1.7 & 1.7 \\
Elongation $\kappa_{95}$ & 0.19 & 0.33 \\
Triangularity $\delta_{95}$ & 39 & 40 \\
Additional power & 1.8 & 1.8 \\
$\mathrm{P}_{\text {add }}[\mathrm{MW}]$ & 1.0 & 1.0 \\
$\beta_{\mathrm{N}}$ & $3.0(2.8)$ & 3 \\
$\mathrm{H}_{98 \mathrm{y}, 2}$ & 0.32 & 10 \\
$\mathrm{q}_{95}$ & $12-13$ & 400 \\
$\mathrm{Q}_{\mathrm{DT}}$ & & \\
$\mathrm{P}_{\text {fusion }}[\mathrm{MW}]$ & & \\
\hline
\end{tabular}

duration (which implies overcoming the present MHD and impurity limit), it could generate in the order of $13 \mathrm{MW}$ of fusion power similar as in 1997 but during a much longer time; longer than the typical time-scales of the various plasma processes. It should be mentioned though that the allowable divertor temperature could restrict the duration of this regime. Nevertheless, development of such a stationary regime is a vital requirement for ITER. The ultimate performance of JET cannot be fully predicted as new territory will be explored.

The progress in the plasma performance that has been achieved with deuterium plasmas in JET after 1997 and the recent enhancements with the ITER-like wall will allow going beyond the 1997 record of highest fusion energy. A simple reproduction of the 1997 regimes could be demonstrated for more extended time and at higher input power. It should be stressed that this is a very challenging and ambitious objective. Indeed, up to now (after the 2014 experimental campaign) the highest fusion performance phase is limited in duration due to a deleterious interplay between the core MHD limits and the core impurity influx and as well by the divertor temperature limit and high $\mathrm{W}$ concentration in the core that are reached in low fuelling conditions. The D-T projections of the 2014 achieved highest D-D neutrons rates scenarios predict respectively 3.2 and 5.5 MW of fusion power for the baseline and hybrid scenarios, i.e. a factor 2-3 with respect to the high level D-T objective. The future challenge in the coming experimental campaigns for preparing D-T operation is to combine a good core confinement with the divertor constraints. The main challenge remains to increase the confinement at higher field/current and applied powers in stationary conditions while not exceeding the temperature 
conditions imposed by the initially cooled W-divertor with acceptable low W concentration in the core. The start of nuclear operation in ITER will be separated from the 1997 D-T experiment on JET by at least 30 years, one generation! Another D-T experiment on JET, roughly a decade in advance of ITER's scientific exploitation will be ideal to prepare the ITER scientists and engineers. The JET D-T experiments will be the final opportunity to train ITER scientists and engineers for the use of tritium before ITER starts its D-T campaign.

\section{JET as International ITER Test Bed}

Experience on JET has taught us that it takes 4 years before the team in the control room is fully functional. Rather than going through this learning curve at ITER, it is much more efficient to train the ITER team at JET to not waste valuable experimental time at ITER.

On this basis it is being investigated whether JET can be extended until about 2025 (see Fig. 1) to train the international team of ITER operators, session leaders and technicians, in addition to performing the very important D-T experiments for ITER. The basic idea is that JET will on the one hand be run by an international team (comprised of scientists, engineers and technicians coming from all ITER partners) that will move to Cadarache to operate ITER at a later stage, and on the other hand be used exclusively to run experiments that help to mitigate any risks in the ITER research plan. To make this possible the ITER IO Physics Team and the international partners will together decide on the International JET research plan. The final D-T experiments in JET could be foreseen around about 2024, which is not long before ITER will come into operation. This is a not to be underestimated benefit, as all knowledge that will be gained will still be fresh and can be readily transferred, along with the trained operators from JET to ITER.

Of course in case JET is extended by such a long period (the presently approved schedule would foresee closure of JET in 2018 - see Fig. 1), maintenance and upgrade tasks are necessary to make sure that all JET systems operate reliably. At the same time it is envisaged to enhance JET with Electron Cyclotron Resonance Heating (ECRH) Systems and Resonant Magnetic Perturbation coils such that plasma scenarios can be studied that are even closer to those expected in ITER. An ECRH system can be used for core MHD control (like NTM), W-control and also to mimic alpha heating. It might be an essential ingredient to access the advanced regime (Table 2) that had to be dropped from the upcoming JET campaign due to lack of non-inductive current drive capabilities at high density operation requires from the $\mathrm{W}$-divertor compatibility. In the extended and internationalised JET schedule, the D-T campaigns would follow between 2023 and 2024 after the installation of enhancements in 2019-2020 and their subsequent testing in a deuterium campaign. With the extended capabilities JET operation will continue to be strongly focused on the consolidation of the physics basis of the three main ITER scenarios (ELMy H-mode, the hybrid scenario and the advanced steady-state scenario) in conditions even closer to ITER thanks to higher electron heating, lower core particle fuelling and lower external torque injection together with the possibility of studying the consequences of ELM mitigation on fusion performance with a metallic wall for ITER. In addition, by increasing the non-inductive current drive fraction, it widens the operational space for developing the stationary regimes in particular with access to a wider range of safety factor profiles. The emphasis remains on the physics extrapolation of the JET results towards ITER by extending the accessible JET operating space.
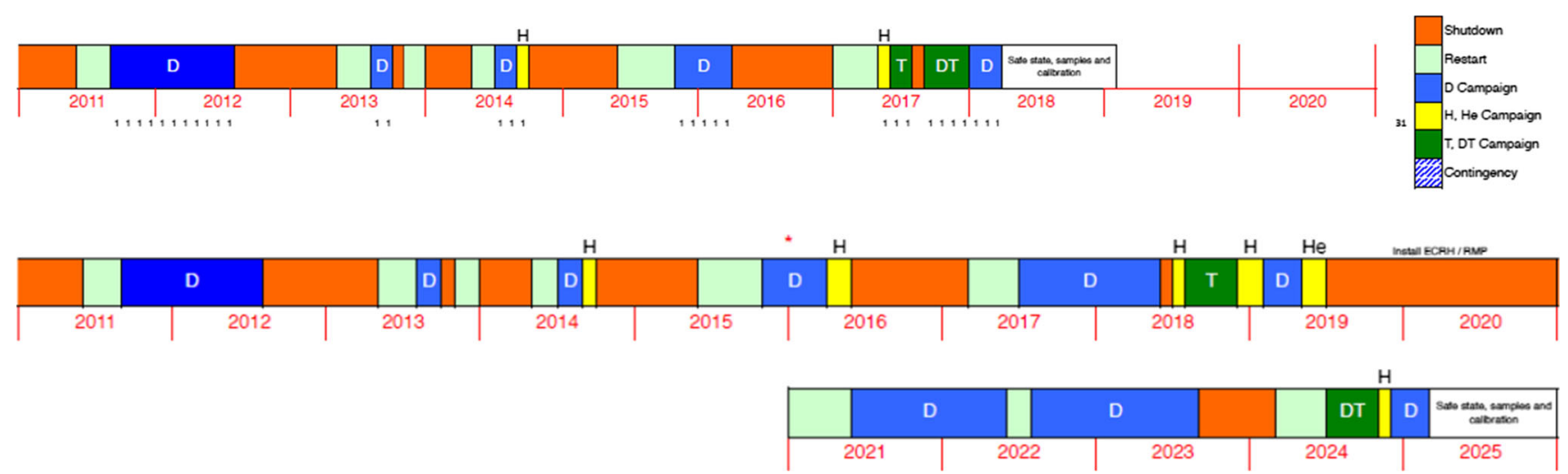

Fig. 1 Officially approved JET schedule with foreseen closure at the end of 2018 (top) and extended time schedule of JET until 2025. A decision on enhancements to be installed in the 2019-2020 shutdown needs to be taken by the end of 2015 in order to have all preparations ready in time. The time schedule after 2020 is indicative and needs to be agreed with all partners that will be involved in JET 
Table 2 Effect of the various campaign options on the risk mitigation for ITER

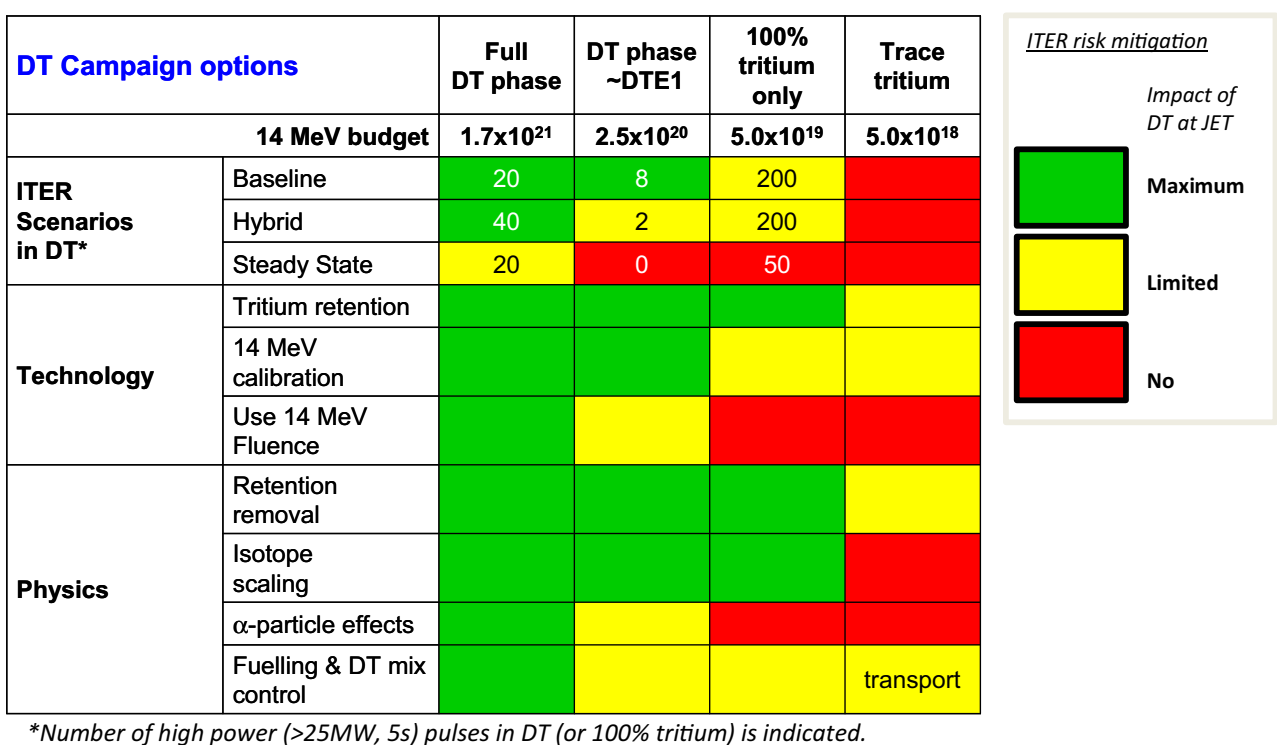

The scientific benefit of the extended schedule is that it provides much more experimental time to develop reliable and high performance scenario in deuterium prior to D-T experiments, making it possible to address ITER physics issues in relevant conditions to mitigate the risks for the active and non-active phases in the ITER research plan [disruption physics and mitigation, ELM physics and mitigation, Plasma-Wall Interaction physics, H-mode and access conditions to high confinement, MHD stability control, experiments covering the entire range of working gases foreseen for ITER (H, D, T and He), etc.]. The main challenge with the metallic wall remains to increase the confinement at higher field/current and applied powers, while reaching stationary conditions compatible with the $\mathrm{W}$-divertor. In addition, with the proposed upgrades, the JET scenarios and the physics scaling experiments could be developed in conditions even closer to ITER conditions thus aiming towards minimising the risk on ITER operation. A full isotope scaling, including T-T operation, is foreseen already in 2018 and 2019, before the enhancements phase. Apart from the scientific value of the isotope scaling, the tritium operation will be important to study tritium retention and also the efficiency of tritium retention removal. Additionally, it will be extremely useful to train engineers on the operation of the tritium plant including the waste processing. In addition the new water detritiation system can be tested. The impact of the $\mathrm{T}-\mathrm{T}$ and the full $\mathrm{D}-\mathrm{T}$ campaign on mitigating risks in the ITER research plan are indicated in Table 2. The most viable path for ITER to access the Type I ELMy H-mode during the nonactive phase is thought to be in helium plasmas. This requires experimental confirmation of the key open issues that affect the operability of ITER in helium $\mathrm{H}$-modes with a W-divertor. For this purpose a He campaign is foreseen shortly after the full T-T campaign in 2019.

\section{JET Enhancements}

A number of enhancements for JET have been studied in detail and in case a positive decision is taken on extending JET under an international framework they would greatly enhance the experimental possibilities of JET and make it possible to even better mimic ITER. The two most sizeable of these enhancements are the installation of an Electron Cyclotron Resonance Heating (ECRH) system and the implementation of Resonant Magnetic Perturbation (RMP) coils.

\section{Electron Cyclotron Resonance Heating}

A study to assess the feasibility of installing an ECRH system on JET has concluded that such a system would significantly improve JET's ability to fulfil its role in preparing ITER operational scenarios [9]. The principal goals of an ECRH system on JET are: current drive over a range of radii for NTM stabilization, sawtooth control and current profile tailoring and central electron heating to equilibrate electron and ion temperatures in high performance discharges and additionally to avoid $\mathrm{W}$ accumulation. The feasibility study [10] concluded that a 12 gyrotron, $10 \mathrm{MW}$, system at the ITER frequency $(170 \mathrm{GHz})$ adapted for fields of $2.7-3.3 \mathrm{~T}$ would be appropriate for the planned operation in JET. It is proposed to use the ITER upper launcher steering mechanism to allow for toroidal and poloidal steering over a wide range. 
ITER diamond windows and transmission line technology can be used and tested while power supply solutions partially reusing existing JET power supplies are proposed. The detailed planning shows that such a system can be operational in about 5 years from the time that the decision to proceed is taken. The cost and required manpower associated with implementing such a system on JET has also been estimated.

ECRH does not produce neutrons directly (as is the case for NBI through beam-target reaction) and neither does it directly heat the ions (which provide the thermal fusion). This makes it difficult to evaluate the effect of ECRH electron heating on the fusion performance, as one has to consider the indirect effects. Firstly, there is a potential increase in the electron temperature, which can have knock-on effects on the ion temperature through equipartition, transport and stability modifications, and the beamtarget reactions through the fast ion slowing-down time (although the latter is of diminishing importance in high temperature plasmas because the electron drag on the NBI injected fast ions is already relatively small). Secondly, ECRH has an impact on the accessible operating-space. If on-axis ECRH can mitigate the effects of high-Z impurity accumulation, it might be possible to reduce the level of gas injection, which is presently one of our tools for controlling the high- $Z$ impurities. This could help to prolong the high performance phase, but would not be applicable at the highest values of magnetic field and current. In the high beta hybrid domain an addition challenge for extending the duration of the high fusion power phase is the need to avoid performance degrading MHD instabilities. In this case one could also consider the use of ECRH for NTM control either by local deposition or optimisation of the overall q-profile shape. This should provide access to higher beta operation by reducing the deleterious MHD modes for the high performance operation at high field (as highlighted in Table 1). For central electron heating, high performance hybrid or baseline scenarios at slightly lower toroidal fields will also be developed. Part of the optimisation, will consist of finding the optimum magnetic field for increasing the fusion performance while maintaining the ECRH system in the safe operational domain (i.e. avoiding far offaxis resonance).The rational for choosing, the ITER ECRH frequency $(170 \mathrm{GHz})$ is based on the possibility of covering the toroidal field range most routinely used on JET and to make the JET ECRH system much more valuable for reducing technical risks for ITER.

In addition, the application of EC waves to JET advanced scenarios has been investigated and modelled using integrated modelling codes. It consists in using offaxis ECCD to produce a significant change of the q-profile [10]. The simulation of an existing JET discharge (\#77895 at $\mathrm{n}_{\mathrm{e} 0} \sim 5.510^{19} \mathrm{~m}^{-3}, \mathrm{I}_{\mathrm{p}}=1.7 \mathrm{MA}$ ) where ECRH power has been added, have shown that $10 \mathrm{MW}$ are sufficient for a local inversion of the q-profile at mid-radius and for fully non-inductive conditions $\left(\mathrm{V}_{\text {loop }}=0\right)$ corresponding to an ECRH driven current of $\sim 200 \mathrm{kA}$ [11]. The location of the peak can be controlled by tilting the poloidal angles. The scenario optimisation will consist in finding the trade-off in plasma density, plasma current and ECRH non-inductive current to reach fully non-inductive conditions compatible with the ITER like wall.

\section{Resonant Magnetic Perturbation Coils}

Erosion and damage caused by Edge Localized Modes (ELMs) is a major hurdle on the route towards achieving magnetic fusion in a reactor scale machine. Scaling predicts that the ELM energy in ITER, if not mitigated, will exceed the acceptable level by a factor of $\sim 20$. Presently, the most promising method of completely suppressing ELMs is to apply resonant magnetic field perturbations (RMP) in the plasma edge. This technique was discovered on DIII-D [11], and experimentation continues on DIII-D, ASDEX Upgrade and several other tokamaks. Because of the importance of controlling ELMs, a set of RMP coils has been designed for ITER [12], based on empirical criteria developed on DIII-D. According to these criteria, the magnetic perturbation spectrum, characterized by poloidal mode number $\mathrm{m}$ and toroidal mode number $\mathrm{n}$ should have a peak near the resonance with the pitch of the magnetic field lines at the plasma edge, i.e. $m=n q(\psi)$, with sufficient amplitude to generate overlapping islands. Non-resonant parts of the spectrum appear detrimental and are to be minimized. However, the validity of these criteria has not been confirmed by other experiments, and a sound physics basis has not yet been established. A system of RMP coils in JET will provide additional information towards our understanding of ELM control by RMP, and extend the dataset for extrapolation towards ITER like plasmas. An RMP system on JET will also extend the experimental possibilities of the JET machine, and is essential in developing ITER relevant scenarios.

A system of two rows of in-vessel coils is proposed as it not only can achieve the empirical island overlap (Chirikov parameter) criteria for ELM suppression as developed on DIII-D, but also permits variation of the spectra allowing for comparison with other experiments and to investigate the physics of ELM suppression. The two rows of coils are positioned above the machine midplane on the low field side as space exists in this region (see Fig. 2). The lower row has 24 coils, which allows for toroidal mode numbers up to 12 and for fine adjustment of the phasing relative to the upper row of 8 coils. The coils are enclosed in vacuum tight cans, extending to the feedthrough, and are cooled by radiation to the vessel wall. The current in the coils is 


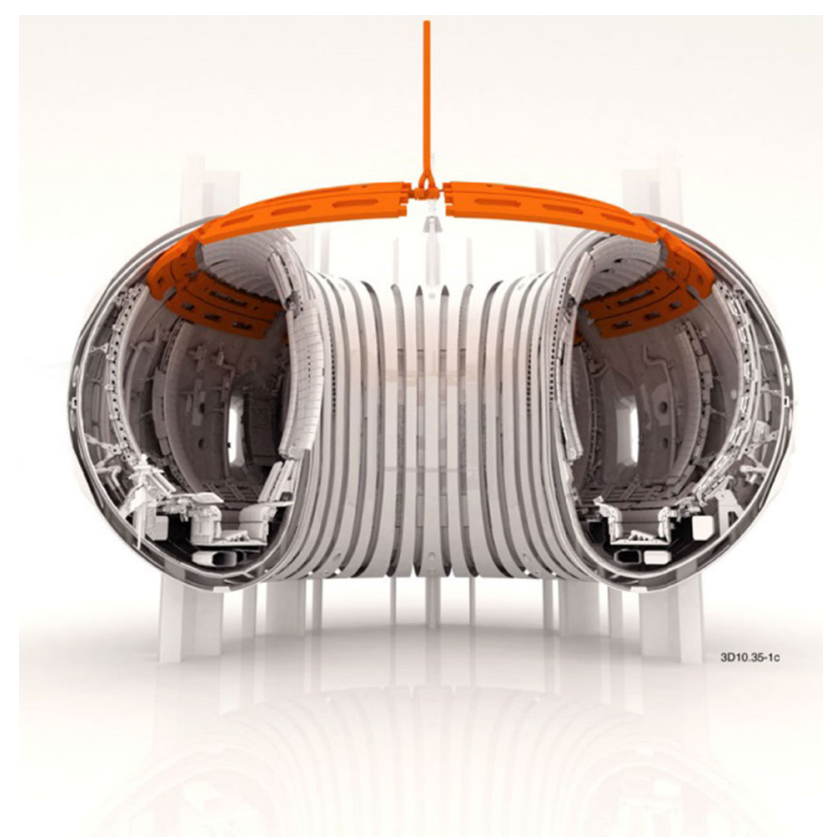

Fig. 2 Artist's impression of Resonant Magnetic Perturbation coils mounted in JET

limited by structural considerations to about $60 \mathrm{kAt}$, which is sufficient to meet the island overlap criteria over the entire JET operational space for low triangularity plasmas, but with some restrictions at high triangularity. At full performance, a pulse repetition rate of two $8 \mathrm{~s}$ pulses per hour is possible in steady state, being dictated by the temperature limit of the polyimide insulator and the weight limit imposed by the remote handling equipment.

\section{Other Enhancements}

Along with the implementation of an ECRH and an RMP system on JET, a suite of diagnostic enhancements is needed to fully support the physics exploitation of the new tools. Specifically, in combination with the RMP enhancement, the JET magnetic diagnostics and control systems need to be upgraded to be compatible with 3D fields. Ideally the new systems to be implemented on JET have the same operating system as is envisaged for ITER, such that the operators and technicians can already get acquainted with these systems.

A tool that could be indispensable for disruption mitigation in ITER and that, ideally, should be tested in JET is a shattered pellet injector [13]. Such a system has been successfully demonstrated on DIII-D as a fast shutdown technique to mitigate the consequences of a disruption, and much can be learned from a demontsration and optimization of this technique on a device that is closer in size to ITER.
Additionally, after 8 years of operation in the present configuration, it will be needed to upgrade or (at least) refurbish the JET divertor to be ready for another 5 years of operation.

In preparation of the ITER scientific exploitation, an important challenge consists in the development of the integrated modelling and analysis suite. The scientific challenges consist of merging our present scientific knowledge into a reliable set of validated simulation tools, accessible and useful for ITER prediction and interpretation activity. Indeed, ITER is a nuclear facility and its exploitation will require systematic

1. Predictive modelling of each discharge from beginning to end, including analysis of real time control requirements, and,

2. Interpretative analysis of each plasma to evaluate and further validate the various models.

This approach of preparing the ITER operation could already be tested in JET with minimum set of software enhancement where the foreseen ITER integrated modelling and analysis suite could be implemented and tested for the discharge preparation and data validation. Various approaches exist among the ITER partners and could be tested for the preparation of the future JET D-T campaign. In this context, EU has developed a standardized platform and an integrated modelling suite of validated numerical codes for the simulation and prediction of a complete plasma discharge [14]. The backbone of the system is a physics- and workflow-oriented data structure which allows for the deployment of a fully modular and flexible workflow organisation. The data structure is designed to be generic and can be used to address physics simulation results, experimental data (including description of subsystem hardware) and engineering issues. In the context of the JET internationalisation, the international Task Forces who will operate JET could already be trained to use the softwares foreseen for ITER exploitation.

\section{Ready for the Next Decade}

After all enhancements, JET will be ready for at least another 5 years of operation under operational conditions that are even closer to ITER. As may be evident from Fig. 1, the emphasis will be on as much as possible operation after the enhancement phase. For the organization of the JET experimental campaigns it is proposed to set up international task forces that operate under the ITER umbrella. It is the ITER project that should be involved in determining the research priorities of JET to ensure that the device is used as adequately as possible for mitigating risks in the ITER research plan. An idea is here to form an 
international JET Governing Board, involving the ITER Physics Team and all international partners that want to join this endeavour.

Although it cannot be denied that other contemporary fusion devices have particular features that will be important to mitigate some of the risks in the ITER research plan (e.g. the super-conducting long pulse devices will yield very useful information on operation of cryogenic devices and any issue related to long pulses; closely monitoring a new device that is taken into operation like JT-60SA will give important lessons to the ITER team), JET has many specific features that make it extremely worthwhile in a risk mitigation strategy for ITER. To summarize the special features of JET:

- It is the largest tokamak device with a full metal wall, and the only one with an ITER-like wall with W divertor and Be tiles;

- It is the only device until ITER that can operate with tritium and with D-T mixtures;

- It has a Be handling facility, an active gas handling facility for tritium and additionally it has a reprocessing plant for tritiated waste;

- It is almost fully remote handling compatible;

- It is already operated by an international team (27 EU countries are directly participating and additional visiting scientists from other Parties are involved) and the experimental campaigns are already organized by international (European) task forces.

It may be evident that after the enhancements mentioned in the previous sections JET will become even more special. Apart from the value it has for ITER, it also gives the opportunity for non-European ITER partners to get involved into real thermonuclear fusion experiments well before ITER will offer this possibility.

The extension of JET until 2025 will have as additional benefit that there is much more time to tackle a number of other high priority topics for ITER. These include a.o.:

- A helium campaign. ITER is planning to access the type-I ELMy H-mode in helium to demonstrate H-mode access and test ELM mitigation control schemes, such to ensure a fast and risk-controlled path to early DT operation (in the officially approved JET schedule there is no time for this, in the extended schedule He-operation is foreseen in 2019).

- Validation of the currently assumed ITER full noninductive steady state scenario. For this objective JET could be used to demonstrate access to high confinement and stability and explore the possibility of profile maintenance assisted by external CD, to test MHD stability at high $\beta$ using resonant field amplification probing, study the influence of ILW-compatible plasma edge conditions on high performance AT scenarios. Additionally advanced tokamak similarity experiments involving JET (metal wall) and JT-60 SA (carbon wall) will help to prepare JT-60SA operation.

- Study novel disruption mitigation methods as Shattered Pellet Injection as well as studying the generation and suppression of runaway electrons.

\section{What is Needed?}

For Europe to turn the idea of extending and internationalizing JET into reality, two important elements are needed. Firstly, this needs to become a project that is organized and run by the international community (i.e. the ITER partners, but possibly also other non-EU partners). Partly this can be done by in-kind contributions for the various enhancements and partly by making people available for the operations team. It is important that these people are long term seconded to JET such that they get a thorough training and are then ready to operate ITER. Secondly, it is important to use JET to build a global ITER physics team, that involves scientists in all the ITER Parties: JET can be used to not only train the scientists, technicians and engineers and to run specific experiments as risk mitigation for ITER but, moreover, it can be also used to test the organizational structure that will be put in place at ITER to organize the experimental campaigns (this should involve international task forces and possibly also an international Governing Board).

In summary, it can be stated that an extension of JET, if organized in the proper way, will be extremely valuable for reducing risks in the ITER research plan, while at the same time preparing the international community for operation of ITER.

Acknowledgments The authors would like to thank C. Challis, J. Garcia, G. Giruzzi, J. Hobirk, L. Horton, M. Lennholm, I. Nunes, E. Solano and for the very useful input and stimulating discussion. This work has been carried out within the framework of the EUROfusion Consortium and has received funding from the Euratom research and training programme 2014-2018 under grant agreement No 633053. The views and opinions expressed herein do not necessarily reflect those of the European Commission.

Open Access This article is distributed under the terms of the Creative Commons Attribution 4.0 International License (http://crea tivecommons.org/licenses/by/4.0/), which permits unrestricted use, distribution, and reproduction in any medium, provided you give appropriate credit to the original author(s) and the source, provide a link to the Creative Commons license, and indicate if changes were made. 


\section{References}

1. O. Motojima et al., The ITER project construction status, 25th IAEA fusion energy conference, St. Petersburg (2014)., paper OV/1-2

2. M. Keilhacker et al., High fusion performance from deuteriumtritium experiments in JET. Nucl. Fusion 39, 209 (1999)

3. L.D. Horton et al., High fusion power steady state operation in JET DT plasmas. Nucl. Fusion 39, 993 (1999)

4. C. Gormezano et al., Internal transport barriers in JET deuteriumtritium plasmas. Phys. Rev. Lett. 80, 5544 (1998)

5. J. Paméla et al., An ITER-like wall for JET. J. Nucl. Mat. 363-365, 1 (2007)

6. F. Romanelli et al., Overview of the JET results, 25th IAEA fusion energy conference, St. Petersburg (2014). Paper OV/1-3

7. P.C. de Vries et al., The influence of an ITER-like wall on disruptions at JET. Phys. Plasmas. 21, 056101 (2014)

8. M. Lehnen et al., Impact and mitigation of disruptions with the ITER-like wall in JET. Nucl. Fusion 53, 093007 (2013)
9. M. Lennholm et al., ECRH for JET: a feasibility study. Fusion Eng. Des. 86, 805 (2011)

10. G. Giruzzi et al., Objectives, physics requirements and conceptual design of an ECRH system for JET. Nucl. Fusion 51, 063033 (2011)

11. T.E. Evans et al., Suppression of large edge localized modes with edge resonant magnetic fields in high confinement DIII-D plasmas. Nucl. Fusion 45, 595 (2005)

12. M.J. Schaffer et al., Study of in-vessel nonaxisymmetric ELM suppression coil concepts for ITER. Nucl. Fusion 48, 024004 (2008)

13. N. Commaux et al., Demonstration of rapid shutdown using large shattered deuterium pellet injection in DIII-D. Nucl. Fusion 50, 112001 (2010)

14. G.L. Falchetto et al., The European integrated tokamak modelling (NTM) effort: achievements and first physics. Nucl. Fusion 54, 043018 (2014) 